Pacific

Journal of

Mathematics

ALEXANDER AND THURSTON NORMS OF FIBERED 3-MANIFOLDS

Nathan M. Dunfield

Volume $200 \quad$ No. 1

September 2001 


\title{
ALEXANDER AND THURSTON NORMS OF FIBERED 3-MANIFOLDS
}

\author{
NATHAN M. DUNFIELD
}

\begin{abstract}
For a 3-manifold $M$, McMullen derived from the Alexander polynomial of $M$ a norm on $H^{1}(M, \mathbb{R})$ called the Alexander norm. He showed that the Thurston norm is an upper bound for the Alexander norm. He asked if these two norms were the same when $M$ fibers over the circle. Here, I give examples that show this is not the case. This question relates to the faithfulness of the Gassner representations of the braid groups. The key tool used is the Bieri-Neumann-Strebel invariant, and I show a connection between this invariant and the Alexander polynomial.
\end{abstract}

\section{Introduction.}

1.1. Statement of results. For a 3-manifold $M$, McMullen derived from the Alexander polynomial of $M$ a norm on $H^{1}(M, \mathbb{R})$ called the Alexander norm. He showed that the Thurston norm on $H^{1}(M, \mathbb{R})$, which measures the complexity of a dual surface, is an upper bound for the Alexander norm. He asked (Question A below) if these two norms were equal on all of $H^{1}(M, \mathbb{R})$ when $M$ fibers over the circle. Here, I will give examples which show that the answer to Question A is emphatically no. As explained below, Question A is related to the faithfulness of the Gassner representations of the braid groups. The key tool used to understand Question A is the Bieri-Neumann-Strebel invariant from combinatorial group theory. Theorem 1.7 below, which is of independent interest, connects the Alexander polynomial with a certain Bieri-Neumann-Strebel invariant.

I will begin by reviewing the definitions of the Alexander and Thurston norms, and Theorem 1.2 which relates them. Then I'll discuss Question A and the connection to the braid groups. After that, I'll state Question B, a much weaker version of Question A, to which the answer is also no. A brief description of the examples which answer these two questions concludes Section 1.1. In Section 1.5, I'll connect these questions with the BieriNeumann-Strebel invariants, and explain why, morally speaking, the answer to both questions must be no. Section 1.8 outlines the rest of the paper.

The Alexander norm is defined in $[\mathbf{M c M}]$ as follows. Let $M$ be a 3manifold (all 3-manifolds in this paper will be assumed to be connected). 
Let $G$ be the fundamental group of $M$. Let $\operatorname{ab}(G)$ denote the maximal free abelian quotient of $G$, which is isomorphic to $\mathbb{Z}^{b_{1}(M)}$ where $b_{1}(M)$ is the first Betti number of $M$. The Alexander polynomial $\Delta_{M}$ of $M$ is an element of the group ring $\mathbb{Z}[\mathrm{ab}(G)]$. It is an invariant of the homology of the cover of $M$ with covering group $\operatorname{ab}(G)$ (for details see Section 3.1). The Alexander norm on $H^{1}(M, \mathbb{R})$ is the norm dual to the Newton polytope of $\Delta_{M}$. That is, if $\Delta_{M}=\sum_{i=1}^{n} a_{i} g_{i}$ with $a_{i} \in \mathbb{Z} \backslash\{0\}$ and $g_{i} \in \operatorname{ab}(G)$ then the norm of a class $\phi \in H^{1}(M, \mathbb{R})$ is defined to be

$$
\|\phi\|_{A}=\sup _{i, j} \phi\left(g_{i}-g_{j}\right) .
$$

The unit ball $B_{A}$ of this norm is, up to scaling, the polytope dual to the Newton polytope of $\Delta_{M}$.

The Thurston norm is defined as follows. For a compact connected surface $S$, let $\chi_{-}(S)=|\chi(S)|$ if $\chi(S) \leq 0$ and 0 otherwise. For a surface with multiple connected components $S_{1}, S_{2}, \ldots, S_{n}$, let $\chi_{-}(S)$ be sum of the $\chi_{-}\left(S_{i}\right)$. Then the Thurston norm of an integer class $\phi \in H^{1}(M, \mathbb{Z}) \cong H_{2}(M, \partial M ; \mathbb{Z})$ is

$$
\begin{gathered}
\|\phi\|_{T}=\inf \left\{\chi_{-}(S) \mid S\right. \text { is a properly embedded oriented surface } \\
\text { that is dual to } \phi\} .
\end{gathered}
$$

As described in [Thu], this norm extends continuously to all of $H^{1}(M, \mathbb{R})$. The unit ball $B_{T}$ in this norm is a finite-sided convex polytope.

It should be noted that both of these "norms" are really semi-normsthey can be zero on non-zero vectors of $H^{1}(M, \mathbb{R})$.

McMullen proved the following theorem which connects the two norms; here $b_{i}(M)=\operatorname{rank} H_{i}(M, \mathbb{R})$ denotes the $i^{\text {th }}$ Betti number of $M$.

Theorem $1.2([\mathbf{M c M}])$. Let $M$ be a compact, orientable 3 -manifold whose boundary, if any, is a union of tori. Then for all $\phi$ in $H^{1}(M, \mathbb{R})$, the Alexander and Thurston norms satisfy

$$
\|\phi\|_{A} \leq\|\phi\|_{T} \quad \text { if } b_{1}(M) \geq 2,
$$

or

$$
\|\phi\|_{A} \leq\|\phi\|_{T}+1+b_{3}(M) \quad \text { if } b_{1}(M)=1 \text { and } \phi \text { generates } H^{1}(M, \mathbb{Z}) .
$$

Moreover, equality holds when $\phi: \pi_{1}(M) \rightarrow \mathbb{Z}$ and $\phi$ can be represented by a fibration $M \rightarrow S^{1}$, where the fibers have non-positive Euler characteristic.

This theorem generalizes the fact that the degree of the Alexander polynomial of a knot is bounded by twice the genus of any Seifert surface. In many simple cases, e.g. almost all the exteriors of the links with 9 or fewer crossings, the Alexander and Thurston norms agree on all of $H^{1}(M, \mathbb{R}$ ) (see $[\mathrm{McM}])$. In such cases, this theorem explains D. Fried's observation from 
the 80 's that frequently the shape of the Newton polytope of the Alexander polynomial is dual to that of the Thurston norm ball.

Before stating Question A, I need to discuss the relationship between the Thurston norm and cohomology classes $\phi: \pi_{1}(M) \rightarrow \mathbb{Z}$ which can be represented by fibrations $M \rightarrow S^{1}$. There are top-dimensional faces, called the fibered faces, of $B_{T}$ such that a class $\phi \in H^{1}(M, \mathbb{Z})$ can be represented by a fibration over the circle if and only if $\phi$ lies in the cone over the interior of one of the fibered faces [Thu, §3]. In this context, the last sentence of Theorem 1.2 is equivalent to "Moreover, the two norms agree on classes that lie in the cone over the fibered faces of $B_{T}$ ". The point of this paper is to answer:

Question A (McMullen $[\mathrm{McM}])$. Let $M$ be a compact, orientable 3-manifold whose boundary, if any, is a union of tori. Suppose that $M$ fibers over the circle and that $b_{1}(M) \geq 2$. Do the Alexander and Thurston norms agree on all of $H^{1}(M, \mathbb{R})$ ?

My motivation for studying this question is McMullen's result that a yes answer would imply that the Gassner representations of the pure braid groups are all faithful [McM, §8]. This would answer in the affirmative the important question: Are the braid groups linear, that is, do they have faithful, finite-dimensional, linear representations? Sadly, I will show that the answer to Question A is no in a strong sense. (Note: Since I wrote this paper, Bigelow and Krammer have independently shown that braid groups are linear [Big2, Kra1, Kra2]. Their proofs use a different representation, and it remains unknown whether the Gassner representation is faithful.)

To explain why the answer to Question A is no, let me formulate a weaker version of Question A which will help make clear some of the issues involved. Henceforth, I will assume that $b_{1}(M) \geq 2$. A typical example of $B_{T}$ is given in Figure 1.3.

There is a pair of fibered faces and the rest of the faces are not fibered. Theorem 1.2 tells us that $\|\cdot\|_{A} \leq\|\cdot\|_{T}$ hence that $B_{A} \supseteq B_{T}$. Since the two norms agree on a fibered face $F_{T}$ of $B_{T}$, there is a face $F_{A}$ of $B_{A}$ which contains $F_{T}$. Now, it seems a bit much to expect that if $M$ fibers over the circle then the two norms agree on classes that are far from any fibered face. So it's reasonable to consider:

Question B. Let $M$ be a compact, orientable 3-manifold whose boundary, if any, is a union of tori. Suppose that $M$ fibers over the circle and that $b_{1}(M) \geq 2$. Let $F_{T}$ be a fibered face of $B_{T}$ and $F_{A}$ the face of $B_{A}$ which contains it. Are $F_{T}$ and $F_{A}$ always equal?

Figure 1.4 shows the two possibilities. Note that a yes answer to Question A implies a yes answer to Question B. I will give examples which show that 


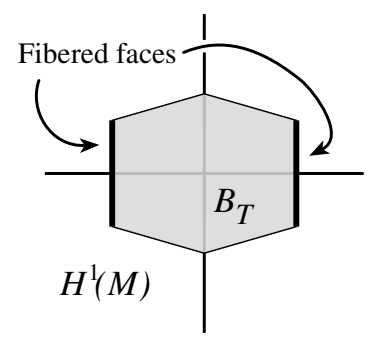

Figure 1.3. The Thurston norm ball.
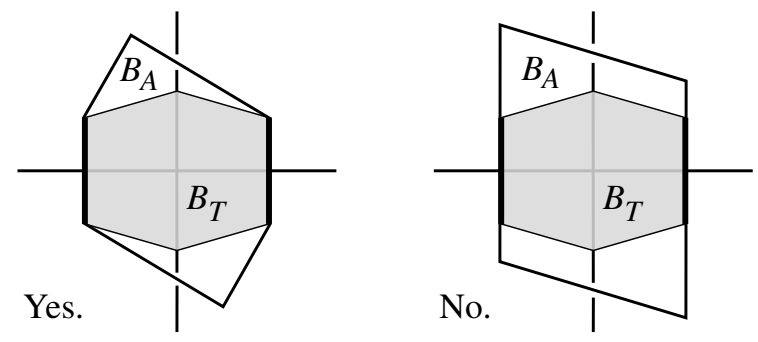

Figure 1.4. Possible answers to Question B.

Answer. The answer to Question B, and therefore Question A, is no.

I will give two kinds of examples. In Section 2, I will constuct examples using the fact that the Burau representation of the braid group on 5 strands is not faithful. Section 2 is independent of the rest of the paper. Section 6 contains an example which is the exterior of a specific 17 crossing link in $S^{3}$.

McMullen's formulation of Question A restricted attention to those manifolds which are the exteriors of links in $S^{3}$. All my examples are such manifolds, but I felt the more general statement was appropriate here.

1.5. Connection to the BNS invariants. In this section I will describe the connection between Question B and the Bieri-Neumann-Strebel (BNS) invariants. In light of this connection, I will explain why the answer to Question B must be, morally speaking, no. The BNS invariants will also be used in constructing and verifying the example in Section 6.

I'll begin with the definition of the BNS invariants (for details see [BNS], and from a different point of view, [Bro]). Let $G$ be a finitely-generated group. Set

$$
S(G)=\left(H^{1}(G, \mathbb{R}) \backslash\{0\}\right) / \mathbb{R}^{+},
$$

where $\mathbb{R}^{+}$acts by scalar multiplication and $S(G)$ is given the quotient topology. A point $[\chi]$ in $S(G)$ will be thought of as an equivalence class of homomorphisms $\chi: G \rightarrow \mathbb{R}$. For $[\chi] \in S(G)$ define $G_{\chi}=\chi^{-1}([0, \infty))=$ $\{g \in G \mid \chi(g) \geq 0\}$, which is a sub-monoid of $G$. 
Let $H$ be a group acted on by $G$ where $G^{\prime}$ acts by inner automorphisms (e.g. $H=G^{\prime}$ where $G$ acts by conjugation). Then the BNS invariant of $G$ and $H$ is:

$$
\begin{aligned}
\Sigma_{H}=\{[\chi] \in S(G) \mid & H \text { is finitely generated over some } \\
& \text { finitely generated sub-monoid of } \left.G_{\chi}\right\} .
\end{aligned}
$$

It turns out that $\Sigma_{H}$ is always an open subset of the sphere $S(G)$.

Let $M$ be a 3 -manifold, and $G=\pi_{1}(M)$. Set $\Sigma=\Sigma_{G^{\prime}}$. Bieri, Neumann, and Strebel proved the following with the help of Stallings' fibration theorem:

Theorem 1.6 ([BNS, Thm. E]). Let $M$ be a compact, orientable, irreducible 3-manifold. Then $\Sigma$ is exactly the projection to $S(G)$ of the interiors of the fibered faces of the Thurston norm ball $B_{T}$.

For convenience, in the rest of this section I will assume that $H_{1}(M, \mathbb{Z})$ is free. This is not essential, and the theory will be developed without this assumption in Sections 3-5. The commutator subgroup $G^{\prime}$ is the fundamental group of the universal abelian cover of $M$. So $A=G^{\prime} / G^{\prime \prime}$ is the first homology of that cover. Thought of as a module over $\mathbb{Z}[\operatorname{ab}(G)], A$ is the Alexander invariant of $M$, from which the Alexander polynomial is derived. Thus it is not too surprising that the BNS invariant $\Sigma_{A}$ is connected to the Alexander polynomial:

Theorem 1.7. Let $M$ be a compact, orientable 3-manifold. There are topdimensional faces $F_{1}, F_{2}, \ldots, F_{n}$ of the Alexander ball $B_{A}$ such that the projection of the interiors of the $F_{i}$ into $S(G)$ is exactly $\Sigma_{A}$. Moreover, the $F_{i}$ are completely determined by the Alexander polynomial of $M$.

Theorem 5.1 below is an expanded version of Theorem 1.7 which explains how the $F_{i}$ are determined. Now since $A$ is a quotient of $G^{\prime}$, it follows immediately from the definitions that $\Sigma_{A} \supset \Sigma$. Combining this with Theorem 1.7, it follows that Question B is equivalent to:

Question $\mathbf{B}^{\prime}$. Let $M$ be a compact, orientable 3-manifold whose boundary, if any, is a union of tori. Suppose that $M$ fibers over the circle and that $b_{1}(M) \geq 2$. Let $C$ be a connected component of $\Sigma$. If $D$ is the connected component of $\Sigma_{A}$ which contains $C$, is $D$ always equal to $C$ ?

Put this way it begins to become clear that the answer to Question B should be no. For many groups $G, \Sigma_{G^{\prime}}$ is strictly contained in $\Sigma_{G^{\prime} / G^{\prime \prime}}$. It remains only to produce examples of 3-manifolds whose fundamental groups have this property.

1.8. Outline of rest of paper. Section 2 describes how to construct examples using the Burau representation. Section 3 defines the Alexander polynomial and proves a fact about the Alexander invariant that's needed 
to prove Theorem 1.7. Section 4 discusses the BNS invariants and records the properties that will be needed later. Section 5 proves the full version of Theorem 1.7. Finally, Section 6 gives an example of a specific link exterior in $S^{3}$ for which the answer to Question B is no.

Acknowledgments. I wrote this paper while a graduate student at the University of Chicago supported by a Sloan Dissertation Fellowship. I would like to thank Curt McMullen for useful correspondence. I got interested in the connection between the Alexander polynomial and Thurston norm at a problem session at KirbyFest (MSRI, June 1998), where Fried's observation was related by Joe Christy. I would like to thank the organizers, MSRI, and the NSF for support to attend that conference. I would also like to thank the referee for help clarifying the exposition.

\section{Connection with braid groups.}

Let $B_{n}$ denote the $n$-strand braid group. McMullen showed that if the answer to Question A is yes, then the Gassner representation of $B_{n}$ is faithful for all $n[\mathbf{M c M}]$. In this section, I'll give a very similar argument to show:

Proposition 2.1. If the answer to Question B is yes, then the Burau representation of $B_{n}$ is faithful for all $n$.

Since the Burau representation of $B_{n}$ is not faithful for $n \geq 5$ [Big1, LP, Moo], the proposition implies that the answer to Question B, and hence Question A, is no.

Before proving the proposition, let me define the braid groups and the Burau representation (see [Bir] for more). Let $D_{n}$ be the disc with $n$ punctures. Consider the group of homeomorphisms $\operatorname{Hom}^{+}\left(D_{n}, \partial D_{n}\right)$ of $D_{n}$ which are orientation preserving and fix $\partial D_{n}$ pointwise. The braid group $B_{n}$ is $\mathrm{Hom}^{+}\left(D_{n}, \partial D_{n}\right)$ modulo isotopies which pointwise fix $\partial D_{n}$.

To define the Burau representation, consider the homomorphism

$$
\phi: H_{1}\left(D_{n}\right) \rightarrow \mathbb{Z}=\langle t\rangle
$$

which takes any clockwise oriented loop about a single puncture to $t$. Let $\widetilde{D}_{n}$ be the cover of $D_{n}$ corresponding to $\phi$. The homology of $\widetilde{D}_{n}$ is a module over the group ring $\mathbb{Z}[\langle t\rangle]$ of the group of covering transformations. The module $H_{1}\left(\widetilde{D}_{n}, \mathbb{Z}\right)$ is free of rank $n-1$. The Burau representation is a homomorphism Burau: $B_{n} \rightarrow \operatorname{Aut}\left(H_{1}\left(\widetilde{D}_{n}\right)\right)$. By Aut $\left(H_{1}\left(\widetilde{D}_{n}\right)\right)$, I mean automorphisms of $H_{1}\left(\widetilde{D}_{n}\right)$ as a $\mathbb{Z}[\langle t\rangle]$-module. Choosing a $\mathbb{Z}[\langle t\rangle]$ basis of $H_{1}\left(\widetilde{D}_{n}\right)$ allows one to view the Burau representation as having image in $\mathrm{GL}(n-1, \mathbb{Z}[\langle t\rangle])$. Given $\beta$ in $B_{n}, \operatorname{Burau}(\beta)$ is constructed as follows. Let $f: D_{n} \rightarrow D_{n}$ be a representative of $\beta$. Choose a lift $\widetilde{f}: \widetilde{D}_{n} \rightarrow \widetilde{D}_{n}$ of $f$. Since the action of $f$ on $H_{1}\left(D_{n}\right)$ commutes with $\phi$, the lift $\tilde{f}$ is equivariant. Thus 
there is a unique lift of $f$ which leaves the inverse image of $\partial D_{n}$ pointwise fixed. Let $\widetilde{f}$ be that lift and set $\operatorname{Burau}(\beta)=\widetilde{f}_{*}: H_{1}\left(\widetilde{D}_{n}\right) \rightarrow H_{1}\left(\widetilde{D}_{n}\right)$.

I'll need the following property of the Burau representation (see also [Mor]). Suppose $\beta$ is a braid whose action on the set of punctures is an $n$-cycle. Let $M_{\beta}$ be the 3 -manifold which is the mapping torus of $\beta$. The manifold $M_{\beta}$ has two boundary components, and $H_{1}\left(M_{\beta}\right)=\mathbb{Z} \oplus \mathbb{Z}$. Take as a basis of $H_{1}\left(M_{\beta}\right)$ the pair $\left(t^{\prime}, w\right)$ where $t^{\prime}$ is a counter-clockwise loop about a puncture in $D_{n}$ and $w$ is a point in $\partial D_{n}$ cross $S^{1}$. It's not hard to see that the universal abelian cover of $M_{\beta}$ is $\widetilde{D}_{n} \times \mathbb{R}$. The covering transformation corresponding to $t^{\prime}$ is $(\widetilde{d}, r) \mapsto(t(\widetilde{d}), r)$, and the covering transformation corresponding to $w$ is $(\widetilde{d}, r) \mapsto(\widetilde{f}(\widetilde{d}), r+1)$. If we replace $t$ by $t^{\prime}$ in $\operatorname{Burau}(\beta)$, the matrix $(w I-\operatorname{Burau}(\beta))$ is a presentation matrix for the homology of the universal abelian cover of $M_{\beta}$ as a $\mathbb{Z}\left[H_{1}\left(M_{\beta}\right)\right]$-module. Thus

$$
\Delta_{M_{\beta}}=\operatorname{det}(w I-\operatorname{Burau}(\beta)) .
$$

I will now prove the proposition.

Proof of Proposition 2.1. Suppose the answer to Question B is yes and the Burau representation of $B_{n}$ has kernel for some $n$. As the Burau representation is known to be faithful for $n=2$, assume $n$ is at least 3. Then there is a pseudo-Anosov element $\delta$ in the kernel [Lon, Iva]. Replacing $\delta$ with a power of $\delta$ if necessary, we can assume $\delta$ is a pure braid, that is, fixes each puncture. Let $\gamma$ be the braid $\sigma_{1} \sigma_{2} \ldots \sigma_{n-1}$ where $\sigma_{i}$ is the $i^{\text {th }}$ standard generator of $B_{n}$ (see Figure 2.2).

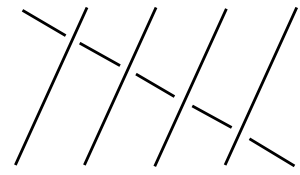

Figure 2.2. The braid $\gamma$ when $n=5$.

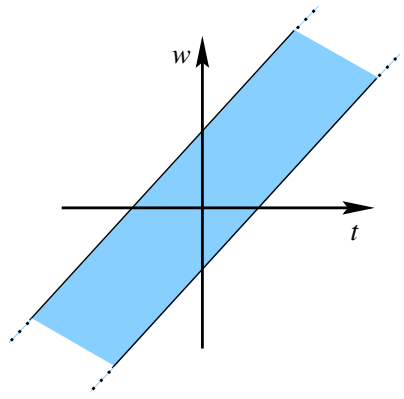

Figure 2.3. The Thurston norm ball of $M_{\gamma}$.

Taking a power of $\delta$ if necessary, we can assume that $\beta=\delta \gamma$ is pseudoAnosov. Now $\beta$ induces an $n$-cycle on the punctures because $\delta$ was a pure braid and $\gamma$ induces an $n$-cycle. Since $\operatorname{Burau}(\beta)=\operatorname{Burau}(\gamma)$, the Alexander polynomials of $M_{\beta}$ and $M_{\gamma}$ are the same. The manifold $M_{\gamma}$ is Seifert fibered, 
and it's easy to see that the Thurston norm ball is as shown in Figure 2.3, where the two infinite faces are fibered faces. Thus by Theorem 1.2, the Alexander norm ball of $M_{\gamma}$ has exactly the same shape as the Thurston norm ball. Since $M_{\gamma}$ and $M_{\beta}$ have the same Alexander polynomials, the Alexander norm ball of $M_{\beta}$ is as shown. But $M_{\beta}$ is hyperbolic, and hence the Thurston norm is non-degenerate. So any face of the Thurston norm ball is bounded. Thus a fibered face of the Thurston norm ball of $M_{\beta}$ is properly contained in the corresponding face of the Alexander norm ball. This contradicts the assumption that the answer to Question B is yes.

\section{The Alexander polynomial and its friends.}

3.1. Definitions. I'll begin by reviewing the definition of the Alexander polynomial and related invariants (for more see [Hil, Rol, McM]). Let $X$ be a finite $\mathrm{CW}$-complex with fundamental group $G$. Let $\tilde{X}$ be the universal free abelian cover of $X$, that is, the cover induced by the homomorphism from $G$ to its free abelianization $\operatorname{ab}(G)$. Let $p$ be a point of $X$, and $\widetilde{p}$ its inverse image in $\widetilde{X}$. The Alexander module of $X$ is

$$
A_{X}=H_{1}(\tilde{X}, \widetilde{p} ; \mathbb{Z})
$$

thought of as a module over the group ring $\mathbb{Z}[\operatorname{ab}(G)]$. The reason one uses the free abelianization is so that the ring $\mathbb{Z}[\operatorname{ab}(G)]$ has no zero divisors.

For a finitely generated module $M$ over $\mathbb{Z}[\operatorname{ab}(G)]$, the $i^{\text {th }}$ elementary ideal $E_{i}(M) \subset \mathbb{Z}[\mathrm{ab}(G)]$ is defined as follows. Take any presentation

$$
0 \rightarrow(\mathbb{Z}[\operatorname{ab}(G)])^{r} \stackrel{P}{\rightarrow}(\mathbb{Z}[\operatorname{ab}(G)])^{s} \rightarrow M \rightarrow 0
$$

and set $E_{i}(M)$ to be the ideal generated by the $(s-i, s-i)$ minors of the matrix $P$. The Alexander ideal of $X$ is $E_{1}\left(A_{X}\right)$. The Alexander polynomial of $X$, denoted $\Delta_{X}$, is the greatest common divisor of the elements of the Alexander ideal. The polynomial $\Delta_{X}$ is defined up to multiplication by a unit $g \in \operatorname{ab}(G)$ of $\mathbb{Z}[\operatorname{ab}(G)]$. Equivalently, $\Delta_{X}$ is a generator of the smallest principle ideal containing the Alexander ideal.

I should mention that the Alexander module, and hence Alexander polynomial, depends only on the fundamental group of $X$; it can be thought of as an invariant of a finitely generated group.

I will need to consider $B_{X}=H_{1}(\widetilde{X} ; \mathbb{Z})$, the Alexander invariant of $X$. When $H_{1}(X ; \mathbb{Z})$ is free, $B_{X}=G^{\prime} / G^{\prime \prime}$. As with $A_{X}$, the Alexander invariant $B_{X}$ is to be thought of as a module over $\mathbb{Z}[\operatorname{ab}(G)]$. The two modules are related as follows. Let $m \subset \mathbb{Z}[\mathrm{ab}(G)]$ be the augmentation ideal, that is $m=\langle 1-g \mid g \in \operatorname{ab}(G)\rangle$. The homology long exact sequence for the pair $(\widetilde{X}, \widetilde{p})$ gives rise to the short exact sequence

$$
0 \rightarrow B_{X} \rightarrow A_{X} \rightarrow m \rightarrow 0 .
$$


The Alexander polynomial of $X$ could just have well been defined as the gcd of $E_{0}\left(B_{X}\right)$ (for the equivalence of these two definitions see, e.g. [Tra]).

3.2. Structure of the Alexander invariant of a 3-manifold. The following fact about the structure of the Alexander ideal of a 3-manifold was crucial in McMullen's proof of Theorem 1.2.

Theorem 3.3 ([McM, 5.1]). Let $M$ be a compact, orientable 3-manifold whose boundary, if any, is a union of tori. Let $G=\pi_{1}(M)$. Then $E_{1}\left(A_{M}\right)=$ $m^{p} \cdot\left(\Delta_{M}\right)$ where

$$
p= \begin{cases}0 & \text { if } b_{1}(M) \leq 1, \\ 1+b_{3}(M) & \text { otherwise, }\end{cases}
$$

and $m$ is the augmentation ideal of $\mathbb{Z}[\mathrm{ab}(G)]$.

The corresponding fact about $E_{0}\left(B_{M}\right)$ will be key to the proof of Theorem 1.7. For a manifold with non-empty torus boundary, Crowell and Strauss $[\mathbf{C S}]$ showed that $E_{0}\left(B_{M}\right)=\left(\Delta_{M}\right) \cdot m^{q}$ for an explicit value of $q$. The following proposition is weaker than $[\mathbf{C S}]$, but it also applies to closed 3-manifolds. It will suffice for my purposes and follows easily from known results.

Proposition 3.4. Let $M$ be a compact, orientable 3-manifold whose boundary, if any, is a union of tori. Then

$$
\sqrt{E_{0}\left(B_{M}\right)} \cap m=\sqrt{\left(\Delta_{M}\right)} \cap m .
$$

Proof. By Theorem 1.1 of [Tra] the short exact sequence

$$
0 \rightarrow B_{M} \rightarrow A_{M} \rightarrow m \rightarrow 0
$$

implies that there are integers $r, s \geq 0$ such that

$$
E_{1}\left(A_{M}\right) \cdot m^{r} \subset E_{0}\left(B_{M}\right) \text { and } E_{0}\left(B_{M}\right) \cdot m^{s} \subset E_{1}\left(A_{M}\right) .
$$

Combining and multiplying by $m$ gives

$$
E_{1}\left(A_{M}\right) \cdot m^{r+s+1} \subset E_{0}\left(B_{M}\right) \cdot m^{s+1} \subset E_{1}\left(A_{M}\right) \cdot m .
$$

Taking radicals of the above and using that $\sqrt{I \cdot J}=\sqrt{I} \cap \sqrt{J}$ gives

$$
\sqrt{E_{1}\left(A_{M}\right)} \cap \sqrt{m}=\sqrt{E_{0}\left(B_{M}\right)} \cap \sqrt{m} .
$$

Now $m$ is radical since it is the kernel of the ring homomorphism $Z[\operatorname{ab}(G)] \rightarrow$ $\mathbb{Z}$ which sends every $g \in \mathrm{ab}(G)$ to 1 . By Theorem 3.3 we have $E_{1}\left(A_{M}\right)=$ $\left(\Delta_{M}\right) \cdot m^{p}$. Combining, we get $\sqrt{E_{0}\left(B_{M}\right)} \cap m=\sqrt{\left(\Delta_{M}\right)} \cap m$ as desired. 


\section{Bieri-Neumann-Strebel invariants.}

Recall the definition of the BNS invariant from Section 1.5. Let $G$ be a finitely-generated group. Let $S(G)=\left(H^{1}(G, \mathbb{R}) \backslash\{0\}\right) / \mathbb{R}^{+}$. For $[\chi] \in$ $S(G)$ we have the sub-monoid $G_{\chi}=\{g \in G \mid \chi(g) \geq 0\}$. Let $H$ be a group acted on by $G$ where $G^{\prime}$ acts by inner automorphisms. Then the BNS invariant of $G$ and $H$ is:

$$
\begin{aligned}
\Sigma_{H}=\{[\chi] \in S(G) \mid & H \text { is finitely generated over some } \\
& \text { finitely generated sub-monoid of } \left.G_{\chi}\right\} .
\end{aligned}
$$

We can also consider the larger invariant

$$
\Sigma_{H}^{\prime}=\left\{[\chi] \in S(G) \mid H \text { is finitely generated over } G_{\chi}\right\} .
$$

When $H$ is abelian $\Sigma_{H}^{\prime}=\Sigma_{H}$ [BNS, Theorem 2.4]. The special case of $\Sigma_{H}^{\prime}$ when both $G$ and $H$ are abelian was studied by Bieri and Strebel [BS] prior to the development of the full BNS invariant. The rest of this section will be devoted to that special case.

Let $Q$ be a finitely generated free abelian group and $A$ a finitely generated $\mathbb{Z}[Q]$-module. Since $A$ has an action of $Q$, we can form the BNS invariant $\Sigma_{A}=\Sigma_{A}^{\prime}$. To reduce clutter, I'll denote $\mathbb{Z}[Q]$ by $\mathbb{Z} Q$. A basic property shown in [BS, $\S 1.3]$ is that $\Sigma_{A}=\Sigma_{\mathbb{Z} Q / \operatorname{Ann}(A)}$ where $\operatorname{Ann}(A)$ is the annihilator ideal of $A$. Thus $\Sigma$ can be seen as an invariant of an ideal $I \subset \mathbb{Z} Q$. The following basic identities hold for any ideals $I, J$ in $\mathbb{Z} Q$ [BS, $\S 1.3]$ :

$$
\Sigma_{\mathbb{Z} Q / I}=\Sigma_{\mathbb{Z} Q / \sqrt{I}} \quad \text { and } \quad \Sigma_{\mathbb{Z} Q /(I \cdot J)}=\Sigma_{\mathbb{Z} Q /(I \cap J)}=\Sigma_{\mathbb{Z} Q / I} \cap \Sigma_{\mathbb{Z} Q / J} .
$$

For principle ideals $I$, the invariant $\Sigma_{\mathbb{Z Q / I}}$ can be easily calculated, as I will now describe. For $p \in \mathbb{Z} Q$, the Newton polytope $\operatorname{Newt}(p)$ is defined as follows. Consider the vector space $V=Q \otimes \mathbb{R}$ which contains $Q$ as a lattice. The Newton polytope of $p$ is the convex hull in $V$ of those $q \in Q$ which have non-zero coefficient in $p$. The vertices of $\operatorname{Newt}(p)$ lie in $Q$, and I'll define the coefficient of a vertex of $\operatorname{Newt}(p)$ to be the non-zero coefficient of the corresponding term of $p$. Given a $q$ in $Q$, define the open hemisphere $H_{q}$ of $S(Q)$ to be

$$
\{[\chi] \in S(Q) \mid \chi(q)>0\} .
$$

The following theorem allows us to calculate $\Sigma_{\mathbb{Z} Q / I}$ for a principle ideal $I$.

Theorem 4.1 ([BS, 5.2]). Let $Q$ be a finitely generated free abelian group and $p$ an element of $\mathbb{Z} Q$. The connected components of $\Sigma_{\mathbb{Z} Q /(p)}$ are in oneto-one correspondence with the vertices of $\operatorname{Newt}(p)$ whose coefficients are \pm 1 , where such a vertex $v$ corresponds to

$$
C_{v}=\bigcap\left\{H_{v w^{-1}} \mid w \text { is a vertex of } \operatorname{Newt}(p) \text { distinct from } v\right\} \text {. }
$$




\section{BNS invariants and Alexander polynomial of a 3-manifold.}

Let $M$ be a compact, orientable 3-manifold whose boundary, if any, is a union of tori. Let $B_{M}=H_{1}(\widetilde{M}, \mathbb{Z})$ be the Alexander invariant of $M$. Regarding $B_{M}$ as a $\mathbb{Z}\left[\operatorname{ab}\left(\pi_{1} M\right)\right]$ module, we can form the BNS-invariant $\Sigma_{B_{M}}$ which I will denote by $\Sigma_{A}$. In Section 1.5 , I defined $\Sigma_{A}$ in case where $H_{1}(M, \mathbb{Z})$ is torsion free, and that definition was slightly different. In the torsion free case, $B_{M}=G^{\prime} / G^{\prime \prime}$ where $G=\pi_{1}(M)$. Thus only difference between the two definitions is that one is the BNS invariant with respect to $\operatorname{ab}(G)$ and the other $G$. Since $B_{M}$ is abelian and $G^{\prime}$ acts trivially on it, the two definitions agree.

In this section I will prove Theorem 5.1 which computes $\Sigma_{A}$ from the Alexander polynomial $\Delta_{M}$. Before stating Theorem 5.1, I need to discuss the unit ball $B_{A}$ in the Alexander norm.

Consider the Newton polytope $\operatorname{Newt}\left(\Delta_{M}\right)$ in $H_{1}(M, \mathbb{R})$. The Alexander norm on $H^{1}(M, \mathbb{R})$ can be defined as

$$
\|\phi\|_{A}=\sup \left\{\phi(x-y) \mid x, y \in \operatorname{Newt}\left(\Delta_{M}\right)\right\} .
$$

A polytope $P$ is balanced about 0 if it is invariant under $v \mapsto-v$. More generally, $P$ is balanced about a point $p$ if the translate of $P$ by $-p$ is balanced about 0 . Since $M$ is a 3 -manifold, $\Delta_{M}$ is symmetric [Bla], [Tur, 4.5], and hence $\operatorname{Newt}(p)$ is balanced about some point $z_{0}$. Then

$$
\|\phi\|_{A}=\sup \left\{2 \phi\left(x-z_{0}\right) \mid x \in \operatorname{Newt}\left(\Delta_{M}\right)\right\}
$$

and the unit ball in $\|\cdot\|_{A}$ is

$$
B_{A}=\left\{\phi \mid \phi\left(x-z_{0}\right) \leq 1 / 2 \text { for all } x \in \operatorname{Newt}\left(\Delta_{M}\right)\right\} .
$$

Fix a basis of $H_{1}(M, \mathbb{R})$ and identify $H^{1}(M, \mathbb{R})$ with $H_{1}(M, \mathbb{R})$ via the dual basis. Then $B_{A}$ is, after scaling by a factor of 2 , the classical polytope dual of $\operatorname{Newt}\left(\Delta_{M}\right)$ about $z_{0}$.

Duality of polytopes in an $n$-dimensional vector space exchanges faces of dimension $i$ with faces of dimension $n-i-1$ (for more on polytope duals, see $[\operatorname{Br} \varnothing])$. A vertex $v$ of $\operatorname{Newt}\left(\Delta_{M}\right)$ becomes the top-dimensional face

$$
F_{v}=\left\{\phi \mid \phi\left(x-z_{0}\right) \leq 1 / 2 \text { for all } x \in \operatorname{Newt}\left(\Delta_{M}\right) \text { and } \phi\left(v-z_{0}\right)=1 / 2\right\} .
$$

I can now state the theorem that relates $\Sigma_{A}$ and $B_{A}$.

Theorem 5.1. Let $M$ be a compact, orientable 3-manifold whose boundary, if any, is a union of tori. Let $F_{1}, \ldots, F_{n}$ be the top-dimensional faces of $B_{A}$ whose corresponding vertices of $\operatorname{Newt}\left(\Delta_{M}\right)$ have coefficient \pm 1 . Then $\Sigma_{A}$ is exactly the projection to $S\left(\mathrm{ab}\left(\pi_{1} M\right)\right)$ of the interiors of the $F_{i}$.

Proof. Let $Q=\operatorname{ab}\left(\pi_{1}(M)\right)$. I will show:

Lemma 5.2. Let $M$ be as above. Then $\Sigma_{A}=\Sigma_{\mathbb{Z} Q /\left(\Delta_{M}\right)}$. 
Let me now deduce the theorem assuming the lemma. By Theorem 4.1, the components of $\Sigma_{\mathbb{Z} Q /\left(\Delta_{M}\right)}$ correspond to the vertices of $\operatorname{Newt}\left(\Delta_{M}\right)$ whose coefficients are \pm 1 . Such a vertex $v$ corresponds to:

$$
C_{v}=\bigcap\left\{H_{v w^{-1}} \mid w \text { is a vertex of } \operatorname{Newt}(p) \text { distinct from } v\right\},
$$

where $H_{q}$ is the hemisphere $\{[\chi] \in S(Q) \mid \chi(q)>0\}$. To prove the theorem it suffices to show $C_{v}$ is the same as the projection into $S(Q)$ of the interior of the face $F_{v}$ of $B_{A}$ corresponding to $v$. Translate Newt $\left(\Delta_{M}\right)$ so it is balanced about 0 - this doesn't change $C_{v}$ or $\|\cdot\|_{A}$. Now note that the cone over the interior of $F_{v}$ is

$\{\phi \mid \phi(v)>\phi(w)$ for all vertices $w$ of $\operatorname{Newt}(p)$ distinct from $v\}$.

It's easy to see that this cone projects to $C_{v}$ in $S(Q)$. This proves the theorem modulo the lemma. Let's go back and prove the lemma.

Proof of Lemma 5.2. The idea of the proof is that Proposition 3.4 says that $B_{M}$ is close, in some sense, to $\mathbb{Z} Q /\left(\Delta_{M}\right)$. Using the properties in Section 4 , we have (notation changed for clarity):

$$
\Sigma_{A}=\Sigma\left(B_{M}\right)=\Sigma\left(\mathbb{Z} Q / \operatorname{Ann}\left(B_{M}\right)\right)=\Sigma\left(\mathbb{Z} Q / \sqrt{\operatorname{Ann}\left(B_{M}\right)}\right) .
$$

For any finitely generated module $B$ we have $\sqrt{\operatorname{Ann}(B)}=\sqrt{E_{0}(B)}$, and so

$$
\Sigma_{A}=\Sigma\left(\mathbb{Z} Q / \sqrt{E_{0}\left(B_{M}\right)}\right) .
$$

Let $m$ be the augmentation ideal of $\mathbb{Z} Q$. Since $\mathbb{Z} Q / m=\mathbb{Z}$, the invariant $\Sigma_{\mathbb{Z} Q / m}$ is all of $S(Q)$. So for any ideal $I$, we have $\Sigma(\mathbb{Z} Q /(I \cap m))=$ $\Sigma(\mathbb{Z} Q / I) \cap \Sigma(\mathbb{Z} Q / m)=\Sigma(\mathbb{Z} Q / I)$. Thus

$$
\Sigma_{A}=\Sigma\left(\mathbb{Z} Q /\left(\sqrt{E_{0}\left(B_{M}\right)} \cap m\right)\right) .
$$

By Proposition 3.4, $\sqrt{E_{0}\left(B_{M}\right)} \cap m=\sqrt{\left(\Delta_{M}\right)} \cap m$, so

$$
\Sigma_{A}=\Sigma\left(\mathbb{Z} Q /\left(\sqrt{\left(\Delta_{M}\right)} \cap m\right)\right)=\Sigma\left(\mathbb{Z} Q /\left(\sqrt{\left(\Delta_{M}\right)}\right)\right)=\Sigma\left(\mathbb{Z} Q /\left(\Delta_{M}\right)\right),
$$

as required. This completes the proof of the lemma and thus the theorem.

5.3. Comparison of $\Sigma_{G^{\prime}}$ and $\Sigma_{A}$ when the homology is not free. Let $M$ be a 3-manifold and $G$ its fundamental group. In Section 1.5, I discussed the connection between $\Sigma_{G^{\prime}}$ and cohomology classes representing fibrations of $M$ over the circle. This is true independent of whether $H_{1}(M, \mathbb{Z})$ has torsion. In Section 1.5, $\Sigma_{G^{\prime}}$ and $\Sigma_{A}$ were compared under the assumption that $H_{1}(M, \mathbb{Z})$ is free. In this case, it is easy to see $\Sigma_{A} \supset \Sigma_{G^{\prime}}$, because $\Sigma_{A}=\Sigma_{B_{M}}$, and $B_{M}=G^{\prime} / G^{\prime \prime}$ is a quotient of $G^{\prime}$. When $H_{1}(M, \mathbb{Z})$ is not free, the relation $\Sigma_{A} \supset \Sigma_{G^{\prime}}$ is still true, but not immediate since $B_{M}$ is 
a quotient of the kernel of the map $G \rightarrow \mathrm{ab}(G)$, but that kernel properly contains $G^{\prime}$.

The purpose of this subsection is simply to prove the that $\Sigma_{A} \supset \Sigma_{G^{\prime}}$ for any $M$, and so show that the motivation given in Section 1.5 makes sense regardless of whether $H_{1}(M, \mathbb{Z})$ is free.

Proposition 5.4. Let $M$ be a 3-manifold. Then $\Sigma_{A} \supset \Sigma_{G^{\prime}}$.

Proof. Let $N$ be the kernel of the map from $G$ to its free abelianization. It is clear that $\Sigma_{A} \supset \Sigma_{N}$ as the Alexander invariant $B_{M}$ is a quotient of $N$. By Proposition 3.4 of [BNS], $\Sigma_{N}=\Sigma_{G^{\prime}}$ and we are done.

\section{Example of a specific link exterior in $S^{3}$.}

Let $L$ be the link in Figure 6.1.

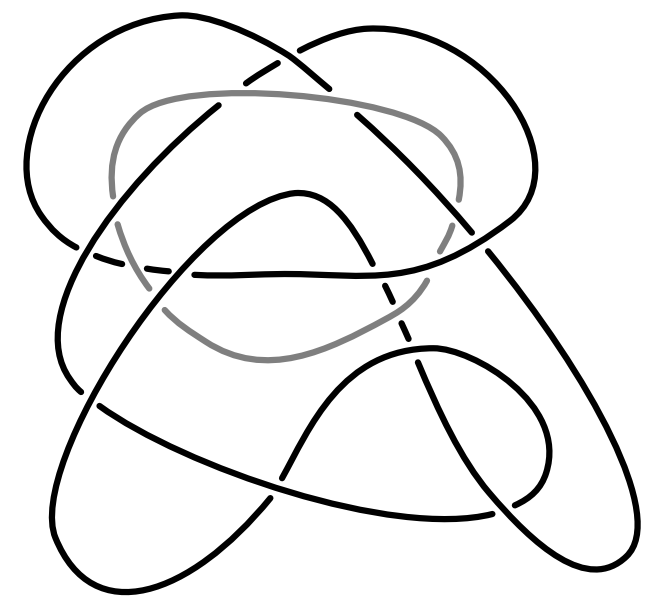

Figure 6.1. The link $L$ in $S^{3}$.

Let $M=S^{3} \backslash N(L)$ be the exterior of $L$. In this section, I'll show that $M$ is a fibered 3 -manifold where the answer to Question B is no. I will do this by explicitly computing the BNS invariants $\Sigma$ and $\Sigma_{A}$, showing that $\Sigma$ is non-empty and that each component of $\Sigma$ is properly contained in the corresponding component of $\Sigma_{A}$. The manifold $M$ is hyperbolic with volume $8.997 \ldots$, as can be checked with the program SnapPea [W], or better, Snap $[\mathbf{G}]$, but I won't use this fact. I found this example by a brute force searchthe program SnapPea was used to find many links whose fundamental groups have a presentation with two generators and one relator. For such groups, it is easy to calculate $\Sigma$ and $\Sigma_{A}$ directly, as I will do below, and eventually I came across this example. 
According to SnapPea, $\pi_{1} M$ has a presentation with two generators $a$ and $b$ and defining relation

$$
\begin{aligned}
& a^{2} b a^{-1} b a^{2} b a^{-1} b^{-3} a^{-1} b a^{2} b a^{-1} b a b^{-1} a^{-2} . \\
& \quad b^{-1} a b^{-1} a^{-2} b^{-1} a b^{3} a b^{-1} a^{-2} b^{-1} a b^{-1} a^{-1} b .
\end{aligned}
$$

A meridian for the unknoted component is $b^{-1} a^{-1} b a^{2} b a^{-1} b a^{2} b a^{-1} b^{-3}$ and a meridian for the other component is $a^{-1} b^{-1}$.

Let $X$ be the 2-dimensional CW-complex corresponding to the above presentation. Let $G=\pi_{1}(X)$. The abelian group $\mathrm{ab}(G)$ is freely generated by images of $a$ and $b$, and so $\mathbb{Z}[\operatorname{ab}(G)]=\mathbb{Z}[\langle a, b\rangle]$.

Let $\widetilde{X}$ be the universal abelian cover of $X$. It is natural to think of the 1 -skeleton of $\widetilde{X}$ as the integer grid in $H_{1}(X, \mathbb{R})$. Let $\delta$ be the lift of $a b a^{-1} b^{-1}$ starting at 0 , which freely generates the 1 -chains of $\widetilde{X}$ as a $\mathbb{Z}[\operatorname{ab}(G)]$ module. The 2-chains of $\widetilde{X}$ are generated by any lift of the 2-cell of $X$. Let $\gamma$ be the lift of the relator to 1 -skeleton of $\widetilde{X}$ starting at 0 , which is homologous in the 1 -skeleton to $\left(a^{2} b-a b-a+1\right) \delta$. Thus

$$
B_{X}=H_{1}(\widetilde{X}, \mathbb{Z})=\mathbb{Z}[\langle a, b\rangle] /\left(a^{2} b-a b-a+1\right) .
$$

So $\Delta_{M}=\Delta_{X}=a^{2} b-a b-a+1$. By Theorem 5.1, or, since $B_{M}$ cyclic, Theorem 4.1 directly, we find that $\Sigma_{A}$ is all of $S(\mathrm{ab}(G))$ except the four points $\left\{ \pm\left[b^{*}\right], \pm\left[a^{*}-b^{*}\right]\right\}$, where $\left\{a^{*}, b^{*}\right\}$ is the dual basis to $\{a, b\}$.

To compute $\Sigma$, I'll use Brown's procedure for computing $\Sigma$ for any group with a 2-generator, 1-relator presentation [Bro, §4]. Think of the 1-skeleton of $\widetilde{X}$ as the integer grid in $H_{1}(X, \mathbb{R})$. Let $C$ be the convex hull of $\gamma$, the lift of the relator. A vertex $v$ of $C$ is called simple if $\gamma$ passes through $v$ only once. Figure 6.2 shows $C$ with the 2 simple vertices $v_{1}$ and $v_{2}$ marked.

Theorem 4.4 of [Bro] shows that in our case $\Sigma$ consists of two components $C_{i}$, for $i=1,2$, where

$$
C_{i}=\bigcap\left\{H_{v_{i} w^{-1}} \mid w \text { is a vertex of } C \text { distinct from } v_{i}\right\} .
$$

Thus $\Sigma$ is the union of the two open intervals pictured in Figure 6.3, and each component of $\Sigma$ is properly contained in the corresponding component of $\Sigma_{A}$. So $M$ shows that the answer to Question B is no. 


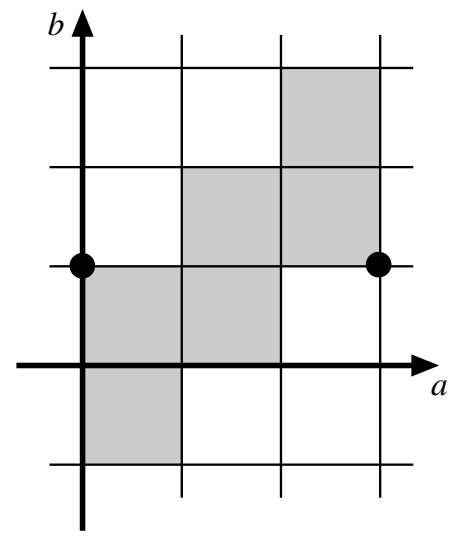

Figure 6.2. The region $C$. The two dots are the simple vertices $v_{1}$ and $v_{2}$.

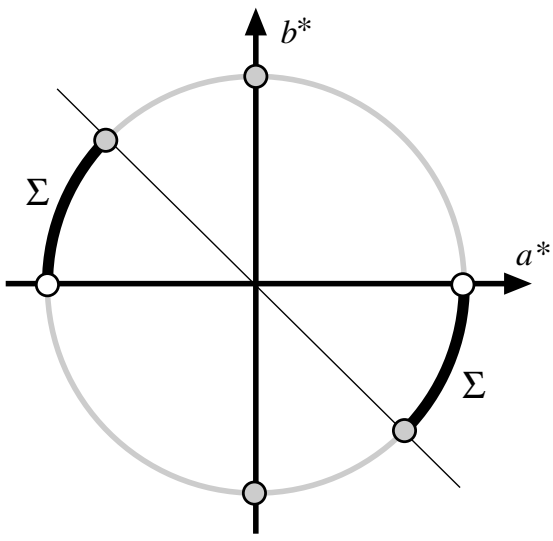

Figure 6.3. $\Sigma \subset S(\mathrm{ab}(G))$ consists of the two open intervals shown. $\Sigma_{A}$ is the complement of the four grey dots.

\section{References}

[BNS] R. Bieri, W.D. Neumann and R. Strebel, A geometric invariant of discrete groups, Invent. Math., 90 (1987), 451-477, MR 89b:20108, Zbl 642.57002.

[BS] R. Bieri and R. Strebel, A geometric invariant for modules over an abelian group, J. Reine Angew. Math., 322 (1981), 170-189, MR 82f:20017, Zbl 448.20010.

[Big1] S. Bigelow, The Burau representation is not faithful for $n \geq 5$, Geometry and Topology, 3 (1999), 397-404, CMP 1725 480, Zbl 942.20017, math/9904100.

[Big2] _ Braid groups are linear, preprint, 2000, math/0005038.

[Bir] J.S. Birman, Braids, Links, and Mapping Class Groups, Princeton University Press, Princeton, N.J., 1974; Annals of Mathematics Studies, 82, MR 54 \#13894, Zbl 305.57013.

[Bla] R.C. Blanchfield, Intersection theory of manifolds with operators with applications to knot theory, Ann. of Math., 65(2) (1957), 340-356, MR 19,53a, Zbl 080.16601.

[Brø] A. Brøndsted, An Introduction to Convex Polytopes, Springer-Verlag, New YorkBerlin, 1983, MR 84d:52009, Zbl 509.52001.

[Bro] K.S. Brown, Trees, valuations, and the Bieri-Neumann-Strebel invariant, Invent. Math., 90 (1987), 479-504, MR 89e:20060, Zbl 663.20033.

[CS] R.H. Crowell and D. Strauss, On the elementary ideals of link modules, Trans. Amer. Math. Soc., 142 (1969), 93-109, Zbl 189.55001.

[G] O. Goodman, Snap; http://www.ms.unimelb.edu.au/〜snap/.

[Hil] J.A. Hillman, Alexander Ideals of Links, Number 895 in Lecture Notes in Math. Springer-Verlag, Berlin-New York, 1981, MR 84j:57004, Zbl 491.57001. 
[Iva] N.V. Ivanov, Subgroups of Teichmüller Modular Groups, American Mathematical Society, Providence, RI, 1992; translated from the Russian by E.J.F. Primrose and revised by the author, MR 93k:57031, Zbl 776.57001.

[Kra1] D. Krammer, The braid group $B_{4}$ is linear, preprint, 1999.

[Kra2] _ The braid groups are linear, preprint, 2000.

[Lon] D.D. Long, A note on the normal subgroups of mapping class groups, Math. Proc. Cambridge Philos. Soc., 99 (1986), 79-87, MR 87c:57009, Zbl 584.57008.

[LP] D.D. Long and M. Paton, The Burau representation is not faithful for $n \geq 6$, Topology, 32 (1993), 439-447, MR 94c:20071, Zbl 810.57004.

[McM] C.T. McMullen, The Alexander polynomial of a 3-manifold and the Thurston norm on cohomology, preprint, 1998.

[Moo] J. Moody, The faithfulness question for the Burau representation, Proc. Amer. Math. Soc., 119 (1993), 671-679, MR 93k:57019, Zbl 796.57004.

[Mor] H.R. Morton, The multivariable Alexander polynomial for a closed braid, in 'Lowdimensional topology (Funchal, 1998)', 167-172. Amer. Math. Soc., Providence, RI, 1999, MR 2000e:57025, Zbl 935.57010, math/9803138.

[Rol] D. Rolfsen, Knots and Links, Publish or Perish Inc., Berkeley, Calif., 1976, Mathematics Lecture Series, 7, MR 95c:57018, Zbl 339.55004.

[Thu] W.P. Thurston, A norm for the homology of 3-manifolds, Mem. Amer. Math. Soc., 59 (1986), i-vi and 99-130, MR 88h:57014, Zbl 585.57006.

[Tra] L. Traldi, The determinantal ideals of link modules, I, Pacific J. Math., 101 (1982), 215-222, MR 84h:57004, Zbl 489.57001.

[Tur] V.G. Turaev, The Alexander polynomial of a three-dimensional manifold, Math. USSR Sbornik, 26 (1975), 313-329, MR 52 \#4306, Zbl 315.57001.

[W] J. Weeks, SnapPea, http://www.northnet.org/weeks/.

Received October 22, 1999 and revised July 17, 2000. This work was partially supported by a Sloan Dissertation Fellowship.

Department of Mathematics

HARVARD UNIVERSITY

CAmbridge, MA 02138

E-mail address: nathand@math.harvard.edu 